\title{
Zebrafish behaviour in profile
}

The complexity of brain function makes it difficult to develop simple high-throughput screens to identify drugs that can target nervous system disorders. Two studies have now addressed this problem by using zebrafish larvae to screen for drugs that affect particular behaviours.

In both studies, zebrafish larvae were maintained in 96-well plates and exposed to numerous small molecules with known and unknown biological effects. Schier and colleagues measured several parameters of locomotor behaviour, including the number and duration of resting and waking activity periods. Similarly, Kokel et al. assessed multiple aspects of the stereotypical 'photomotor response' to a light stimulus. Thus, a behavioural 'fingerprint' for each compound that described the compound's specific effects could be obtained.

Clustering algorithms were used to group the molecules according to their effects. As expected, compounds that are known to have similar functional effects, biological targets, chemical scaffolds or therapeutic indications in humans had similar behavioural fingerprints. This suggests that the effects of compounds identified in the zebrafish screen are likely to be conserved in other vertebrates.
The screens can be used used to predict the targets of compounds with unknown mechanisms of action. For example, Schier and colleagues found that the compound MRS-1220 had a fingerprint similar to that of monoamine oxidase (MAO) inhibitors. A subsequent biochemical assay confirmed that MRS-1220 inhibits MAO. Similarly, Kokel et al. found that STR-1 and STR-2 clustered with known acetylcholine esterase (AChE) inhibitors and subsequently confirmed that these compounds inhibited AChE in activity assays.

This approach could also provide new insights into the pathways that regulate particular behaviours. For example, Schier and colleagues found that several inflammatory compounds specifically increased daytime waking, implicating inflammatory signalling pathways in the regulation of daytime activity.

Finally, Kokel et al. showed that their screen could be used to identify compounds that suppress the effects of drugs that target particular neural pathways, which may hold promise for the identification of therapeutic compounds for specific disorders.

These screens enable the rapid assessment of the effects of many compounds on complex, physiologically relevant behaviours. In addition to elucidating the mechanisms of action of existing and novel compounds, similar assays with relevance to specific human behaviours might contribute to the discovery of new therapeutics for neurological and psychiatric disorders.

Katherine Whalley

ORIGINAL RESEARCH PAPERS Rihel, J. et al. Zebrafish behavioural profiling links drugs to biological targets and rest/wake regulation. Science 327, 348-351 (2010) | Kokel, D. et al. Rapid behavior-based identification of neuroactive small molecules in the zebrafish. Nature Chem. Biol. 17 Jan 2010 (doi:10.1038/ nchembio.307)

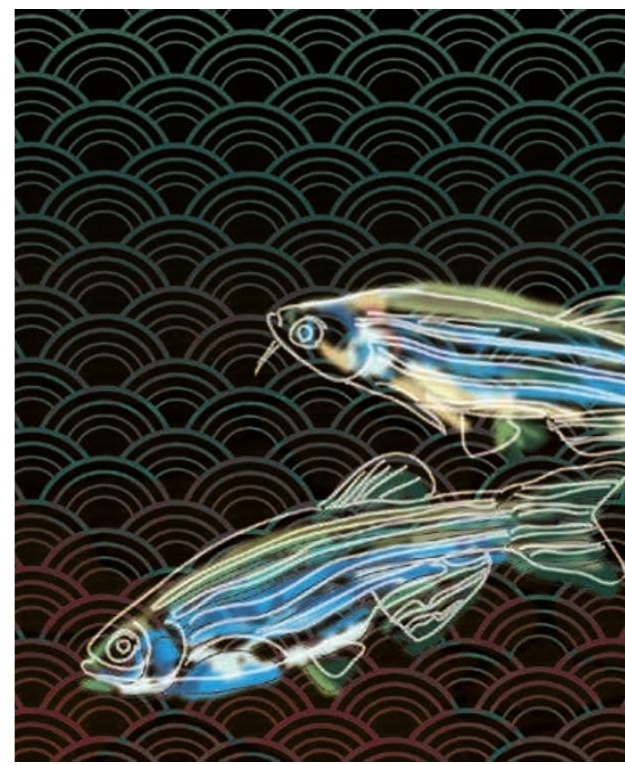

